\title{
Multi-Channel Sensing and Access Game: Bayesian Social Learning with Negative Network Externality
}

\author{
Chunxiao Jiang, Member, IEEE, Yan Chen, Member, IEEE, and K. J. Ray Liu, Fellow, IEEE
}

\begin{abstract}
In a distributed cognitive radio network, due to negative network externality, rational secondary users tend to avoid accessing the same vacant primary channels with others. Moreover, they usually need to make their channel access decisions in a sequential manner to avoid collisions. The characteristic of negative network externality and the structure of sequential decision making make the multi-channel sensing and access problem challenging, which has not been well studied by the existing literatures. To solve these problems, in this paper, we propose a multi-channel sensing and access game, which not only considers the negative network externality in secondary users' decision making, but also takes into account their sequential decision making structure. We solve the multichannel sensing problem using Bayesian learning method and design a cooperative learning rule for secondary users to accurately estimate the channel state. We study the multi-channel access problem under two scenarios: with and without resource constraint, respectively. For both scenarios, we design recursive best response algorithms for secondary users to find the subgame perfect Nash equilibria. Specifically, we analyze the homogenous case of the scenario without resource constraint and find that the Nash equilibrium profile exhibits a threshold structure. Finally, we conduct simulations to validate the effectiveness and efficiency of the proposed methods.
\end{abstract}

Index Terms-Cognitive radio, cooperative spectrum sensing, dynamic spectrum access, Bayesian learning, game theory.

\section{INTRODUCTION}

$\mathbf{R}$ ECENTLY, due to the emergence of various wireless applications, electromagnetic radio spectrums are becoming more and more crowded. Under such circumstances, dynamic spectrum access (DSA) schemes are proposed to mitigate the problem of crowded electromagnetic radio spectrum [1]. Compared with static spectrum allocation, it has been shown to be able to significantly enhance the utilization efficiency of existing spectrum resources [2]. Cognitive radio technology is considered as an effective approach to implement such DSA schemes. In a cognitive radio network, the unlicensed users, called as Secondary Users (SUs), can opportunistically utilize the spectrum resources of licensed users, called as Primary Users (PUs) under the constraint of without harmful interference to the PUs [3].

Manuscript received July 5, 2013; revised October 15, 2013; accepted December 23, 2013. The associate editor coordinating the review of this paper and approving it for publication was W. Zhang.

C. Jiang is with the Department of Electronic Engineering, Tsinghua University, Beijing 100084, P. R. China. This work was done during his visit at the University of Maryland, College Park (e-mail: chx.jiang@gmail.com).

Y. Chen and K. J. R. Liu are with the Department of Electrical and Computer Engineering, University of Maryland, College Park, MD 20742, USA (e-mail: \{yan, kjrliu\}@umd.edu)

This work was partly funded by project 61371079 and 61271267 supported by NSFC China, Postdoctoral Science Foundation funded project.

Digital Object Identifier 10.1109/TWC.2014.022014.131209
Two essential issues of dynamic spectrum access are spectrum sensing and channel access [4]. Due to the uncertainty about the states of multiple primary channels, i.e., whether the PUs are active, SUs need to first estimate the channel state through spectrum sensing [5]. Spectrum sensing methods based on energy detection and waveform sensing were proposed in [6] and [7], respectively. In order to counter the channel fading and shadowing problem, cooperative spectrum sensing technology was proposed, in which SUs share their spectrum sensing results to improve the sensing performance [8]. In [9], the authors proposed a multi-channel cooperative sensing scheme, including slotted-time sensing and continuous-time sensing. After multi-channel sensing, SUs access primary channels for data transmission. Several spectrum access methods based on different mathematical models have been proposed, e.g., Markov decision process (MDP) based approaches [10]-[11], queuing theoretic approaches [12]-[14], and game theoretic approaches [15]-[17]. Moreover, the joint spectrum sensing and channel access problems were studied in [18]-[20].

When making channel access decision, each SU not only should consider the channel quality, but also take into account other SUs' channel access decisions since the more SUs access the same channel, the less throughput each SU can obtain. Such a phenomenon is known as negative network externality [21], i.e., the negative influence of other users' behaviors on one user's reward, due to which each user tends to avoid making the same decision with others to maximize his/her own utility. Moreover, in a fully distributed cognitive radio network, SUs usually need to make decision sequentially to avoid collision, which makes the multiple SUs' multi-channel sensing and access problem even more challenging. Although this is an important and practical issue, there is few work in the literature considering both negative network externality and sequentially decision making structure. In our previous works [22]-[23], we proposed a Chinese Restaurant Game to address the sequential decision making with negative network externality. However, the underlying assumption of Chinese Restaurant Game is that each SU can only access one primary channel at each time slot [24]-[25], due to which it cannot be directly applied to the multi-channel sensing and access problem.

Recently, we proposed a new game, called Indian Buffet Game [26], to study how users in a dynamic system make multiple concurrent decisions on different objects/resources when confronted with uncertain system state. In the Indian Buffet Game, there are finite dishes with different dish qualities and finite customers sequentially requesting multiple 
dishes for meal. Since customers do not know the exact dish quality, i.e., the state of each dish, they have to learn the dish state according to some external information. Moreover, when requesting multiple dishes, a customer needs to take into account the decisions of other customers, especially those of subsequent customers, due to the negative network externality. The multi-channel sensing and access problem in cognitive radio networks can be ideally modeled by the Indian Buffet Game [27], where the SUs can be regarded as customers and the multiple primary channels can be regarded as multiple dishes in the restaurant. Furthermore, how SUs estimate the state of each primary channel can be regarded as how customers learn the state of each dish, and how a SU chooses multiple channels to access can be formulated as how a customer requests multiple dishes for meal.

Based on the Indian Buffet Game model, in this paper, we propose a multi-channel sensing and access game for distributed cognitive radio network by considering both negative network externality and SU's sequential decision making structure. We analyze the proposed game under two scenarios: multi-channel sensing and access with resource constraint and without resource constraint, respectively, where the resource constraint means that each SU can at most sense and access a certain number of channels. For the multi-channel sensing problem, we introduce a cooperative channel state learning method based on Bayesian learning. With this learning scheme, SUs first cooperatively learn the channel utilization ratio, i.e., the prior probability that primary channel is idle, and then construct their belief regarding the channel state of each time slot, i.e., the posterior probability, using both their spectrum sensing results and the estimated channel utilization ratio. For SUs' multi-channel access problem without resource constraint, we show that the multi-channel access game can be decoupled into a series of independent single-channel access game. We then design a recursive best response algorithm to find the subgame perfect Nash equilibrium for SUs. Moreover, we show that the Nash equilibrium under the homogeneous setting exhibits a threshold structure. For the multi-channel access problem with resource constraint, we also design a recursive best response algorithm for SUs to find the subgame perfect Nash equilibrium.

The rest of this paper is organized as follows. Firstly, we introduce the proposed multi-channel sensing and access framework in Section II. Then, we discuss how SUs learn the primary channel state using Bayesian learning rule in Section III. In Section VI and Section V, we analyze the multiple SUs' multi-channel access game without and with resource constraint, respectively. Finally, we show simulation results in Section VI and draw conclusions in Section VII.

\section{Multi-Channel Sensing And Access Framework}

\section{A. System Model}

We consider a primary network with $M$ independent primary channels denoted by $\left\{\mathrm{Ch}_{1}, \mathrm{Ch}_{2}, \ldots, \mathrm{Ch}_{M}\right\}$, as shown in Fig. 1. The PUs have priority to occupy these $M$ channels at any time, while SUs are allowed to access the channels under the condition that the PUs' communication QoS is guaranteed [2]. The primary channels are assumed to be

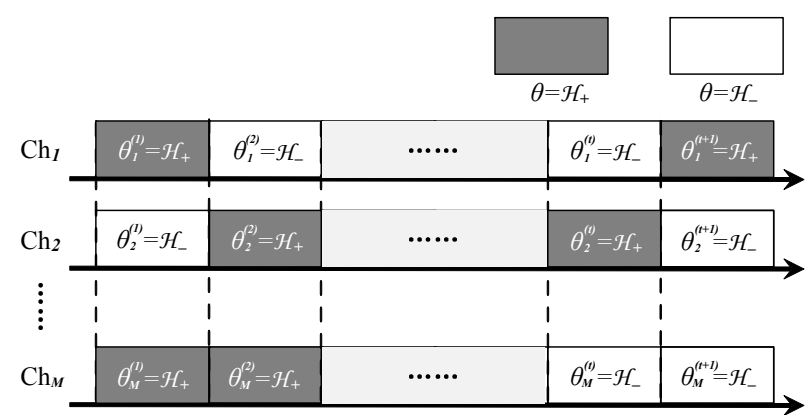

Fig. 1. Primary channels model.

slotted, and there exist idle time slots, during which no PUs' communication appears and SUs have opportunity to access. We denote the primary channel state as $\boldsymbol{\theta}=\left\{\theta_{1}, \theta_{2}, \ldots, \theta_{M}\right\}$, where $\theta_{j} \in\left\{\mathcal{H}_{-}, \mathcal{H}_{+}\right\}^{1}$ represents the state of channel $\mathrm{Ch}_{j}$, $\mathcal{H}_{-}$means the channel is idle and $\mathcal{H}_{+}$means the PUs are active in the channel. Note that the channel state $\theta_{j}^{(t)}$ is timevarying since PUs may appear at the primary channel at any time. We assume that all primary channels are stationary, which means that each channel has a steady channel utilization ratio, denoted by $\lambda_{j}$, where

$$
\left\{\begin{array}{l}
\operatorname{Pr}\left(\theta_{j}^{(t)}=\mathcal{H}_{-}\right)=\lambda_{j}, \\
\operatorname{Pr}\left(\theta_{j}^{(t)}=\mathcal{H}_{+}\right)=1-\lambda_{j},
\end{array} \quad \forall j=1,2, \ldots, M .\right.
$$

In our model, the state $\theta_{j}^{(t)}$ and the utilization ratio $\lambda_{j}$ are unknown to SUs.

For the secondary network, suppose that there are $N$ SUs, labeled by $\{1,2, \ldots, N\}$, searching vacant primary channels for data transmission. The communication in the secondary network is also time slotted and synchronous with that of the primary network. We assume that each SU can simultaneously access multiple vacant primary channels during one time slot, which can be implemented by Orthogonal Frequency Division Multiplexing (OFDM) technology. All SUs are considered as rational users in the sense that each SU makes multichannel access decision with the objective of maximizing his/her own expected reward. Since SUs do not know exactly the instantaneous channel state $\theta_{j}^{(t)}$, they need to listen to the primary channels for a while before channel access. We consider such spectrum sensing as a prerequisite for all secondary devices and each SU can independently perform spectrum sensing using energy detection method [28].

\section{B. SUs' Time Slot Structure}

As shown in Fig. 2, we illustrate the time slot structure in the secondary network. At each time slot $t \in\{1,2 \ldots\}$, there are three phases: channel learning phase, access decision phase and data transmission phase.

In the channel learning phase, SUs cooperatively learn the channel state at current time slot. There are two steps in this phase: utilization ratio learning and channel state learning. In the first step, each SU independently performs spectrum

\footnotetext{
${ }^{1}$ In the paper, the bold symbols represent vectors, the bold capital symbols represent matrixes, the subscript $i$ means the SU index and subscript $j$ means the channel index, the superscript $(t)$ means the time slot index.
} 


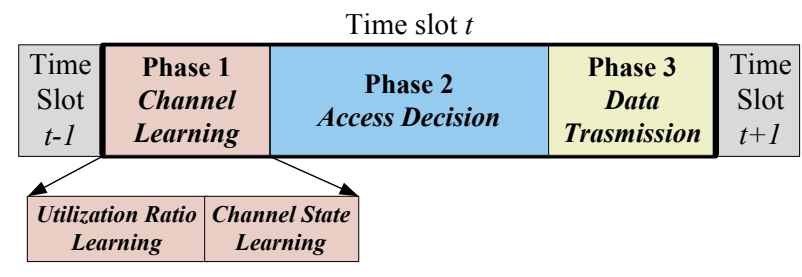

Fig. 2. SUs' time slot structure.

sensing to obtain some observations on whether the primary channels are vacant. Due to resource constraint, each SU is assumed to be able to sense at most $L(L \leq M)$ primary channels at one time slot. According to the observations, SUs cooperatively learn the utilization ratio of each channel, $\lambda_{j}$, which can be regarded as the prior probability of channel state. We assume that there is a narrow-band control channel in the secondary network for SUs to exchange their spectrum sensing information. In the second step, based on the observation from spectrum sensing and the estimation of $\lambda_{j}$, SUs cooperatively estimate the state of each primary channel at the current time slot. Here, we introduce the concept of belief to describe SUs' uncertainty about the current channel state, denoted by

$$
\mathbf{p}^{(t)}=\left\{p_{j}^{(t)}=\operatorname{Po}\left(\theta_{j}^{(t)}=\mathcal{H}_{-}\right), j=1,2, . ., M\right\},
$$

where $p_{j}^{(t)}$ represents SUs' estimation of the probability that channel $\mathrm{Ch}_{j}$ is in idle state at time slot $t$ after performing channel sensing. Notice that $p_{j}^{(t)}$ is the posterior probability of $\theta_{j}^{(t)}=\mathcal{H}_{-}$, which is different from the prior probability $\lambda_{j}=\operatorname{Pr}\left(\theta_{j}=\mathcal{H}_{-}\right)$. The details about the proposed cooperative channel learning scheme will be discussed in Section III.

In the access decision phase, all SUs make decisions on which channels to access to and broadcast their decisions to others via the control channel. In order to avoid the collision, we assume that SUs sequentially make and broadcast their decisions according to a certain predefined order. As we will see later, SUs' behaviors and utilities highly depend on the decision order. For the sake of fairness, we assume that the decisions order is randomized and thus different at different time slot, due to which SUs are uncertain about their future decision orders and only care about the expected reward at current time slot. To maximize the expected rewards, SUs need to consider not only the channel state and previous SUs' decisions, but also the subsequent SUs' decisions. In Section IV and V, we will discuss the best decision of each SU in this multi-channel access problem.

In data transmission phase, after all SUs have made their channel access decisions, each SU begins data transmission on the corresponding channels. If the SUs access a primary channel with active PUs, they will receive little throughput due to the interference from PUs' communications. On the other hand, if SUs successfully access a vacant channel, they will share the channel through Time Division Multiple Access (TDMA) or Code Division Multiple Access (CDMA).

To summarize, we show in Algorithm 1 the proposed multichannel sensing and access framework.

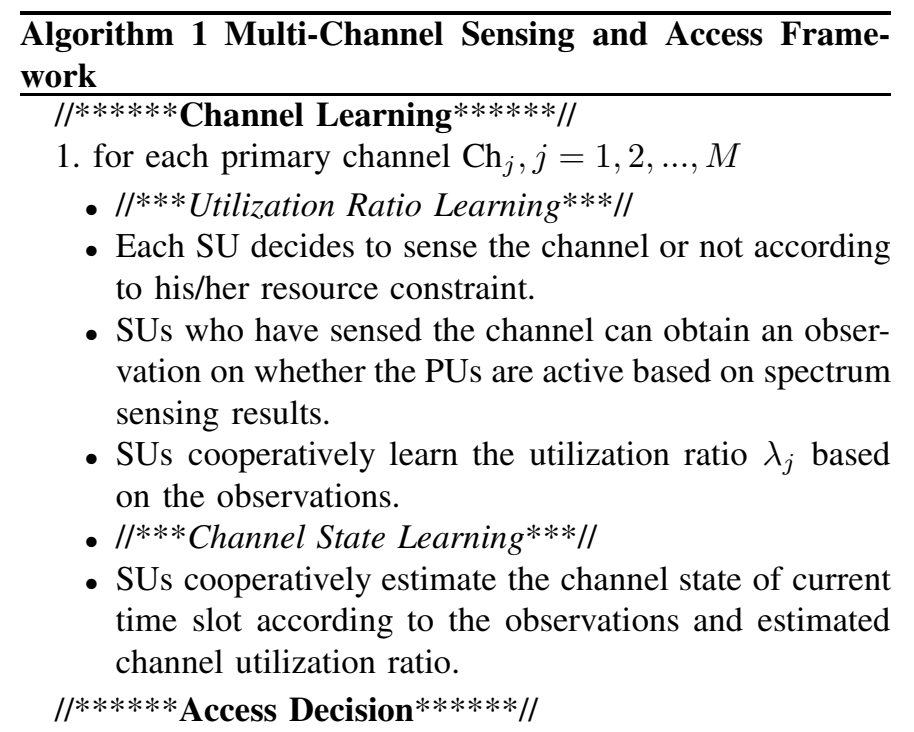

2. for each SU $(i=1,2, \ldots, N)$

- Each SU sequentially decides which channels to access to.

- Each SU broadcasts his/her decisions to other SUs.

$/ / * * * * * *$ Data Transmission $* * * * * * / /$

3. Each SU transmits his/her data in corresponding channels.

\section{Multi-Channel Access Game Formulation}

As discussed above, after channel learning phase, SUs begin to sequentially make decisions on which channels to access to. As rational users, SUs should take into account all possible factors to maximize their expected payoffs, including channel state, i.e., channel is vacant or occupied, as well as previous and subsequent SUs' decisions, i.e., the negative network externality. In this paper, we formulate this multi-channel access problem as a non-cooperative game and derive the best response for each SU. Here, we first define the players, strategies, utilities and best responses of the multi-channel access game model as follows.

- Players: $N$ rational SUs in the secondary network.

- Strategies: Since each SU can access multiple primary channels, the strategy set can be defined as

$$
\mathcal{X}=\left\{\emptyset,\left\{r_{1}\right\}, \ldots,\left\{r_{1}, r_{2}\right\}, \ldots,\left\{r_{1}, r_{2}, \ldots, r_{M}\right\}\right\}
$$

where each strategy is a combination of channels and $\emptyset$ means no channel is accessed. Obviously, SUs' entire strategy set is finite with $2^{M}$ elements. Let us denote the strategy of the $i$-th SU at time slot $t$ as $\mathbf{d}_{i}^{(t)}=$ $\left(d_{i, 1}^{(t)}, d_{i, 2}^{(t)}, \ldots, d_{i, M}^{(t)}\right)^{\prime}$, where $d_{i, j}^{(t)}=1$ represents that the $i$-th SU accesses channel $\mathrm{Ch}_{j}$ at time slot $t$ and otherwise we have $d_{i, j}=0$. In such a case, the strategy profile of 
all SUs can be denoted by a $M \times N$ matrix as follows:

$$
\begin{aligned}
\mathbf{D}^{(t)} & =\left(\mathbf{d}_{1}^{(t)}, \mathbf{d}_{2}^{(t)}, \ldots, \mathbf{d}_{N}^{(t)}\right), \\
& =\left[\begin{array}{cccc}
d_{1,1}^{(t)} & d_{2,1}^{(t)} & \cdots & d_{N, 1}^{(t)} \\
d_{1,2}^{(t)} & d_{2,2}^{(t)} & \cdots & d_{N, 2}^{(t)} \\
\vdots & \vdots & \ddots & \vdots \\
d_{1, M}^{(t)} & d_{2, M}^{(t)} & \cdots & d_{N, M}^{(t)}
\end{array}\right] .
\end{aligned}
$$

- Utility function: Each SU can expect his/her transmission data rate by the belief of the channel state and the number of SUs who will share the same channel with him/her. Assuming SUs share the primary channel through TDMA, we can define the expected utility function of the $i$-th SU accessing channel $\mathrm{Ch}_{j}$ at time slot $t$ as

$$
U_{i, j}^{(t)}=\frac{p_{j}^{(t)} g_{i, j}}{N_{j}^{(t)}}-c_{j},
$$

where $p_{j}^{(t)}$ is SUs' belief of the state of channel $\mathrm{Ch}_{j}$ at time slot $t, g_{i, j}$ is the $i$-th SU's gain of accessing channel $\mathrm{Ch}_{j}$ which depends on the channel gain, $N_{j}^{(t)}$ is the total number of SUs sharing channel $\mathrm{Ch}_{j}$ at time slot $t$ and $c_{j}$ is the cost of accessing channel $\mathrm{Ch}_{j}$. From (5), we can see that the more accurate the belief, the better the expected utility SUs can obtain. Moreover, the utility function is a decreasing function in terms of $N_{j}$, which can be regarded as the characteristic of negative network externality since the more SUs access channel $\mathrm{Ch}_{j}$, the lower utility each SU can obtain.

- Best response: The best response of the $i$-th $\mathrm{SU}, \mathbf{d}_{i}^{(t) *}=$ $\left(d_{i, 1}^{(t) *}, d_{i, 2}^{(t) *}, \ldots, d_{i, M}^{(t) *}\right)^{\prime}$, is to maximize his/her expected utility, which can be written as

$$
\mathbf{d}_{i}^{(t) *}=\underset{\mathbf{d}_{i}^{(t)} \in\{0,1\}^{M}}{\arg \max } \sum_{j=1}^{M} d_{i, j}^{(t)} \cdot U_{i, j}^{(t)} .
$$

Note that due to hardware limitation and/or power constraint, SUs may not be able to access all channels at one time slot, i.e., there may be a resource constraint. In this paper, we assume that each SU can at most simultaneously access L channels at each time slot. In such a case, SUs' decisions are subject to the following constraints:

$$
\sum_{j=1}^{M} d_{i, j}^{(t)} \leq L, \quad \forall i=1,2, \ldots, N,
$$

A special case of (7) is $L \geq M$, which means there is no resource constraint for SUs. In Section IV and V, we will explicitly discuss the multi-channel access game under two scenarios: without resource constraint $(L \geq M)$ and with resource constraint $(L<M)$, respectively.

\section{Multi-Channel Sensing with Cooperative Channel State Learning}

As mentioned above, the state of each primary channel $\theta_{j}^{(t)} \in\left\{\mathcal{H}_{-}, \mathcal{H}_{+}\right\}$is time-varying with a steady utilization ratio $\lambda_{j}$, both $\theta_{j}$ and $\lambda_{j}$ are unknown to all SUs but can influence SUs' behaviors and utilities to a large extent. From
(5), we can see that the more accurate channel state estimation, the better channel access decision each SU can make and thus the higher expected reward each SU can obtain. Therefore, it is important to design an effective learning rule so that SUs can gradually learn the true utilization ratio of each channel and accurately estimate the channel state at every time slot. In this subsection, we will discuss how SUs should estimate the channel state in the multi-channel sensing problem. Specifically, we propose a cooperative channel state learning scheme based on Bayesian social learning for SUs.

Although each SU can sense multiple channels during the channel learning phase, they might not be able to sense all channels due to various kinds of resource constraints, such as hardware constraint, power constraint and/or spectrum sensing time constraint. In such a case, each SU may not be able to update his/her beliefs on some channels at every time slot, which inevitably restricts his/her channels access decisions in the following access decision phase. Thus multiple SUs' cooperative channel state learning becomes highly necessary.

In our model, each SU first randomly selects $L(L \leq M)$ channels for spectrum sensing, and then makes a binary decision on the channel state individually. The binary decision is a binary signal $s \in\left\{s_{+}, s_{-}\right\}$, where the positive signal $s_{+}$ indicates that the channel is vacant, while the negative signal $s_{-}$stands for the channel is occupied by PUs. The binary signal of each $\mathrm{SU}$ follows a distribution that

$$
\begin{aligned}
& f\left(s \mid \theta=\mathcal{H}_{-}\right)=\left\{\begin{array}{lll}
P_{f}, & \text { if } & s=s_{+} \\
1-P_{f}, & \text { if } & s=s_{-}
\end{array}\right. \\
& f\left(s \mid \theta=\mathcal{H}_{+}\right)=\left\{\begin{array}{lll}
P_{d}, & \text { if } & s=s_{+} \\
1-P_{d}, & \text { if } & s=s_{-} .
\end{array}\right.
\end{aligned}
$$

where $P_{d}$ and $P_{f}$ are SUs' detection probability and falsealarm probability, respectively. Note that the values of $P_{d}$ and $P_{f}$ reflect the performance of spectrum sensing and depend on SUs' hardware configuration. In this paper, we assume that $P_{d}$ and $P_{f}$ are known to each SU. After spectrum sensing, all SUs exchange their signals with each other for cooperative channel learning, i.e., hard decision fusion rule [8]. The learning phase includes two steps: utilization ratio learning and channel state learning, which will be discussed in details in the following. Since the channel state learning processes of different primary channels are independent of each other, in the rest of this section, we omit the channel index $j$ for notation simplification.

\section{A. Cooperative Channel Utilization Ratio Learning}

The steady utilization ratio of the channel, $\lambda$, is an important prior information for SUs to estimate current channel state. As defined in (1), $\lambda$ represents the prior probability that the primary channel is in idle state. Suppose $\lambda=\Lambda_{k} \in \boldsymbol{\Lambda}$, where $\boldsymbol{\Lambda}$ is a finite set combining all possible $K$ distributions. When the secondary network is first established, a uniform prior is used, i.e., $P^{(0)}\left(\lambda=\Lambda_{k}\right)=1 / K, \forall \Lambda_{k} \in \boldsymbol{\Lambda}$. After SUs begin to perform spectrum sensing, they can improve the estimation of $\lambda$ based on the spectrum sensing results, i.e., the observed signals. Let us denote $\mathbf{s}^{(t)}=\left\{s_{1}^{(t)}, s_{2}^{(t)}, \ldots, s_{N^{(t)}}^{(t)}\right\}$ as the signal set observed by $N^{(t)}$ SUs who have sensed the channel at time slot $t$. Since all SUs share their signals with others, each SU 
can update the estimation of channel utilization ratio using Bayesian learning rule [29] as

$$
P^{(t)}\left(\lambda=\Lambda_{k}\right)=\frac{P^{(t-1)}\left(\lambda=\Lambda_{k}\right) P\left(\mathbf{s}^{(t)} \mid \lambda=\Lambda_{k}\right)}{\sum_{\Lambda_{k} \in \Lambda} P^{(t-1)}\left(\lambda=\Lambda_{k}\right) P\left(\mathbf{s}^{(t)} \mid \lambda=\Lambda_{k}\right)},
$$

where $P^{(t)}\left(\lambda=\Lambda_{k}\right)$ is the estimated probability that channel utilization ratio is $\Lambda_{k}$ given all observed signals up to time slot $t$, i.e., $\left\{\mathbf{s}^{(1)}, \mathbf{s}^{(2)}, \ldots, \mathbf{s}^{(t)}\right\}$, and $P\left(\mathbf{s}^{(t)} \mid \lambda=\Lambda_{k}\right)$ is the conditional probability of observing $\mathbf{s}^{(t)}$ given channel utilization ratio $\lambda=\Lambda_{k}$.

When given current channel state $\theta^{(t)}$, the utilization ratio $\lambda$ and SUs' observed signal $s_{i}^{(t)}$ are independent of each other, i.e.,

$$
P\left(\mathbf{s}^{(t)}, \theta^{(t)} \mid \lambda\right)=P\left(\mathbf{s}^{(t)} \mid \theta^{(t)}\right) P\left(\theta^{(t)} \mid \lambda\right)
$$

In such a case, we have

$$
\begin{aligned}
P\left(\mathbf{s}^{(t)} \mid \lambda=\Lambda_{k}\right)= & P\left(\mathbf{s}^{(t)}, \theta^{(t)}=\mathcal{H}_{+} \mid \lambda=\Lambda_{k}\right)+ \\
& P\left(\mathbf{s}^{(t)}, \theta^{(t)}=\mathcal{H}_{-} \mid \lambda=\Lambda_{k}\right) \\
= & P\left(\mathbf{s}^{(t)} \mid \theta^{(t)}=\mathcal{H}_{+}\right)\left(1-\Lambda_{k}\right)+ \\
& P\left(\mathbf{s}^{(t)} \mid \theta^{(t)}=\mathcal{H}_{-}\right) \Lambda_{k} .
\end{aligned}
$$

Since all SUs independently perform spectrum sensing, the signals $\left\{s_{1}^{(t)}, s_{2}^{(t)}, \ldots, s_{N^{(t)}}^{(t)}\right\}$ observed by $N^{(t)}$ SUs are independent of each other when given current channel state $\theta^{(t)}$, i.e.,

$$
P\left(\mathbf{s}^{(t)} \mid \theta^{(t)}\right)=\prod_{i=1}^{N^{(t)}} f\left(s_{i}^{(t)} \mid \theta^{(t)}\right) .
$$

In such a case, we have

$$
\begin{aligned}
P\left(\mathbf{s}^{(t)} \mid \theta^{(t)}=\mathcal{H}_{+}\right)= & \prod_{i=1}^{N_{+}^{(t)}} f\left(s_{i}^{(t)}=s_{+} \mid \theta^{(t)}=\mathcal{H}_{+}\right) . \\
& \prod_{i=1}^{N_{-}^{(t)}} f\left(s_{i}^{(t)}=s_{-} \mid \theta^{(t)}=\mathcal{H}_{+}\right) \\
= & P_{d}^{N_{+}^{(t)}}\left(1-P_{d}\right)^{N_{-}^{(t)}}
\end{aligned}
$$

where $N_{+}^{(t)}+N_{-}^{(t)}=N^{(t)}$, and $N_{+}^{(t)}$ and $N_{-}^{(t)}$ represent the number of observed positive signals $s_{+}$and negative signals $s_{-}$, respectively. Similarly, we have

$$
P\left(\mathbf{s}^{(t)} \mid \theta^{(t)}=\mathcal{H}_{-}\right)=P_{f}^{N_{+}^{(t)}}\left(1-P_{f}\right)^{N_{-}^{(t)}} .
$$

Combing (14), (15) and (12), we have

$$
\begin{aligned}
P\left(\mathbf{s}^{(t)} \mid \lambda=\Lambda_{k}\right)= & \left(1-\Lambda_{k}\right) P_{d}^{N_{+}^{(t)}}\left(1-P_{d}\right)^{N_{-}^{(t)}}+ \\
& \Lambda_{k} P_{f}^{N_{+}^{(t)}}\left(1-P_{f}\right)^{N_{-}^{(t)}} .
\end{aligned}
$$

Finally, by substituting (16) into (10), we can summarize the cooperative utilization ratio learning rule in (18).

\section{B. Cooperative Channel State Learning}

Channel utilization ratio $\lambda$ is the probability that the primary channel is vacant, which can be used to estimate the channel state at current time slot, i.e., the posterior probability that the channel is vacant. At the beginning of Section III, we have defined the concept of belief to represent such a posterior probability, which can be denoted by

$$
p^{(t)}=\operatorname{Po}\left(\theta^{(t)}=\mathcal{H}_{-}\right)=P\left(\theta^{(t)}=\mathcal{H}_{-} \mid \mathbf{s}^{(t)}, \lambda\right),
$$

From (19), we can see that when estimating channel state at current time slot, SUs not only consider all signals observed at current time slot $\mathbf{s}^{(t)}$, but also implicitly take into account the signals at all previous time slots through the estimated $\lambda$. Suppose that the utilization ratio estimated by SUs $\lambda=\Lambda_{k}$, we can derive SUs' belief as follows:

$$
\begin{aligned}
\left.p^{(t)}\right|_{\lambda=\Lambda_{k}} & =P\left(\theta^{(t)}=\mathcal{H}_{-} \mid \mathbf{s}^{(t)}, \lambda=\Lambda_{k}\right) \\
& =\frac{P\left(\mathbf{s}^{(t)}, \theta^{(t)}=\mathcal{H}_{-} \mid \lambda=\Lambda_{k}\right)}{P\left(\mathbf{s}^{(t)} \mid \lambda=\Lambda_{k}\right)} \\
& =\frac{\Lambda_{k} P\left(\mathbf{s}^{(t)} \mid \theta^{(t)}=\mathcal{H}_{-}\right)}{P\left(\mathbf{s}^{(t)} \mid \lambda=\Lambda_{k}\right)},
\end{aligned}
$$

where the second equality is based on Bayes' theorem [30] and the third equality is due to the independence between $\lambda$ and $\mathbf{s}^{(t)}$ when given $\theta^{(t)}$. With $\left.p^{(t)}\right|_{\lambda=\Lambda_{k}}$, we can calculate SUs' belief at time slot $t$ by

$$
p^{(t)}=\left.\sum_{\Lambda_{k} \in \boldsymbol{\Lambda}} P^{(t)}\left(\lambda=\Lambda_{k}\right) \cdot p^{(t)}\right|_{\lambda=\Lambda_{k}},
$$

where $P^{(t)}\left(\lambda=\Lambda_{k}\right)$ is updated by (18). Combining (15), (16), (20) and (21), we can summarize the cooperative channel state

Utilization ratio learning rule:

$$
P^{(t)}\left(\lambda=\Lambda_{k}\right)=\frac{P^{(t-1)}\left(\lambda=\Lambda_{k}\right)\left[\left(1-\Lambda_{k}\right) P_{d}^{N_{+}^{(t)}}\left(1-P_{d}\right)^{N_{-}^{(t)}}+\Lambda_{k} P_{f}^{N_{+}^{(t)}}\left(1-P_{f}\right)^{N_{-}^{(t)}}\right]}{\sum_{\Lambda_{k} \in \boldsymbol{\Lambda}} P^{(t-1)}\left(\lambda=\Lambda_{k}\right)\left[\left(1-\Lambda_{k}\right) P_{d}^{N_{+}^{(t)}}\left(1-P_{d}\right)^{N_{-}^{(t)}}+\Lambda_{k} P_{f}^{N_{+}^{(t)}}\left(1-P_{f}\right)^{N_{-}^{(t)}}\right]}, \forall \Lambda_{k} \in \boldsymbol{\Lambda} .
$$

Channel state learning rule:

$$
p^{(t)}=\sum_{\Lambda_{k} \in \Lambda} P^{(t)}\left(\lambda=\Lambda_{k}\right) \cdot \frac{\Lambda_{k} P_{f}^{N_{+}^{(t)}}\left(1-P_{f}\right)^{N_{-}^{(t)}}}{\left(1-\Lambda_{k}\right) P_{d}^{N_{+}^{(t)}}\left(1-P_{d}\right)^{N_{-}^{(t)}}+\Lambda_{k} P_{f}^{N_{+}^{(t)}}\left(1-P_{f}\right)^{N_{-}^{(t)}}} .
$$


learning rule in (22). In Section VI, we will conduct simulation to evaluate the performance of proposed channel state learning method.

\section{Multi-Channel Access Game without RESOURCE CONSTRAINT}

In this section, we study the $N$ SUs' multi-channel access game without resource constraint, which is corresponding to the case where $L \geq M$ in (7). When there is no resource constraint, SUs should access all the primary channels that can give them positive expected utility to maximize their total expected utilities. We will first show that without resource constraint, whether to access one primary channel is independent of other channels, i.e. the multi-channel access game can be decoupled to a series of single-channel access games. Then we present a recursive algorithm that characterize the subgame perfect equilibrium of the $N$ SUs' multi-channel access game without budge constraint. Finally, we discuss the homogeneous case where SUs have the same form of utility function to gain more insights. Note that since we discuss the multi-channel access game in one time slot, the superscript $(t)$ is omitted in Section IV and V.

To show the independence among different channels, we first analyze one SU's best response given other SUs' channel access decisions. Let us define $\mathbf{n}_{-i}=$ $\left\{n_{-i, 1}, n_{-i, 2}, \ldots, n_{-i, M}\right\}$ with

$$
n_{-i, j}=\sum_{\zeta \neq i} d_{\zeta, j}
$$

being the number of SUs accessing channel $\mathrm{Ch}_{j}$ except the $i$-th SU . Let $\mathbf{p}=\left\{p_{1}, p_{2}, \ldots, p_{M}\right\}$, where $p_{j}=\operatorname{Po}\left(\theta_{j}=\mathcal{H}_{0}\right)$ is SUs' belief regarding the state of channel $\mathrm{Ch}_{j}$ at current time slot. Given $\mathbf{p}$ and $\mathbf{n}_{-i}$, according to (6), the $i$-th SU's best response, $\mathbf{d}_{i}^{*}=\left(d_{i, 1}^{*}, d_{i, 2}^{*}, \ldots, d_{i, M}^{*}\right)^{\prime}$, can be written as

$$
\mathbf{d}_{i}^{*}=\mathrm{BR}_{i}\left(\mathbf{p}, \mathbf{n}_{-i}\right)=\underset{\mathbf{d}_{i} \in\{0,1\}^{M}}{\arg \max } \sum_{j=1}^{M} d_{i, j} \cdot U_{i, j}
$$

where $U_{i, j}$ is the $i$-th SU's expected utility of accessing channel $\mathrm{Ch}_{j}$ and given by (5).

Since there is no constraint for the optimization problem in (24) and $U_{i, j}$ is only related with $d_{i, j}$, we can re-write (24) by

$$
\mathbf{d}_{i}^{*}=\mathrm{BR}_{i}\left(\mathbf{P}, \mathbf{n}_{-i}\right)=\sum_{j=1}^{M} \underset{\mathbf{d}_{i} \in\{0,1\}^{M}}{\arg \max } d_{i, j} \cdot U_{i, j},
$$

From (25), we can see that a SUs best decision on one channel is irrelevant to his/her decisions on others, which leads to the independence among different channels. In such a case, we have

$$
d_{i, j}^{*}=\underset{d_{i, j} \in\{0,1\}}{\arg \max } d_{i, j} \cdot U_{i, j}
$$

The independence property enables us to simplify our analysis by breaking the origin multi-channel access game into $M$ single-channel access game. In the remaining of this section, we will focus on the analysis of the $N$ SUs' singlechannel access game and drop the channel index $j$ for notation simplification. As a result, we can rewrite the best response of the $i$-th SU as

$$
\begin{aligned}
d_{i}^{*} & =\mathrm{BR}_{i}\left(p, n_{-i}\right)=\underset{d_{i} \in\{0,1\}}{\arg \max } d_{i} \cdot U_{i} \\
& =\left\{\begin{array}{cc}
1, & \text { if } U_{i}=\frac{p g_{i}}{n_{-i}+1}-c>0 ; \\
0, & \text { otherwise. }
\end{array}\right.
\end{aligned}
$$

where $p$ is SUs' belief regarding the single channel state and $c$ is SUs' single-channel access cost.

\section{A. Recursive Best Response Algorithm}

In this subsection, we study how to solve the best response defined in (27) for each SU. From (27), we can see that the $i$-th SU needs to know $n_{-i}$ to calculate the expected utility $U_{i}$ to decide whether to access the channel or not. Since all SUs make decisions sequentially, the $i$-th SU needs to predict the subsequent SUs' decisions based on the belief and known information.

Let $m_{i}$ denote the number of SUs that decide to access the channel after the $i$-th SU, then we can write the recursive form of $m_{i}$ as

$$
m_{i}=m_{i+1}+d_{i+1}
$$

Let $\left.m_{i}\right|_{d_{i}=0}$ and $\left.m_{i}\right|_{d_{i}=1}$ represent $m_{i}$ under the condition of $d_{i}=0$ and $d_{i}=1$, respectively. Denote by $n_{i}=\sum_{\zeta=1}^{i-1} d_{\zeta}$, the number of SUs choosing the channel before the $i$-th SU. Then, the estimated number of SUs accessing the channel excluding the $i$-th SU can be written as follows:

$$
\begin{aligned}
& \left.\hat{n}_{-i}\right|_{d_{i}=0}=n_{i}+\left.m_{i}\right|_{d_{i}=0}, \\
& \left.\hat{n}_{-i}\right|_{d_{i}=1}=n_{i}+\left.m_{i}\right|_{d_{i}=1} .
\end{aligned}
$$

Note that $\left.\hat{n}_{-i}\right|_{d_{i}=0}$ and $\left.\hat{n}_{-i}\right|_{d_{i}=1}$ are different from $n_{-i}$ in that the values of $d_{i+1}, d_{i+2}, \ldots, d_{N}$ are estimated instead of true observations.

According to (30), we can compute the expected utility of the $i$-th SU if accessing the channel, i.e., when $d_{i}=1$, as

$$
\left.U_{i}\right|_{d_{i}=1}=\frac{p g_{i}}{n_{i}+\left.m_{i}\right|_{d_{i}=1}+1}-c .
$$

Since the utility of the $i$-th SU is zero when $d_{i}=0$, the best response of the $i$-th SU can be obtained by

$$
d_{i}^{*}=\left\{\begin{array}{cc}
1, & \text { if }\left.\quad U_{i}\right|_{d_{i}=1}>0 \\
0, & \text { otherwise }
\end{array}\right.
$$

With (32), we can find the best response of the $i$-th SU given belief $p$, current observation $n_{i}$ and predicted number of subsequent SUs accessing the channel, $\left.m_{i}\right|_{d_{i}=1}$. To predict $\left.m_{i}\right|_{d_{i}=1}$, the $i$-th SU needs to predict the decisions of all SUs from $i+1$ to $N$. When it comes to the $N$-th SU, since he/she knows exactly the decisions of all the previous SUs, he/she can find the best response without making any prediction, i.e., $m_{N}=0$. Based on such an intuition, we design a recursive algorithm to predict $\left.m_{i}\right|_{d_{i}=1}$ by considering all possible decisions of SUs from $i+1$ to $N$ and updating $m_{i}=m_{i+1}+d_{i+1}$. In Algorithm 2, we show the recursive algorithm BR_SCA $\left(p, n_{i}, i\right)^{2}$ that describes how to predict

\footnotetext{
${ }^{2} \mathrm{SCA}$ is abbreviation for single-channel access.
} 
$\left.m_{i}\right|_{d_{i}=1}$ and find the best response $d_{i}$ for the $i$-th SU, given current belief $p$ and observation $n_{i}$. Note that the complexity of Algorithm 2 is determined by the number of SUs $N$. Since the first SU needs to predict all the subsequent SUs' decisions, he/she is with the highest complexity, which is at the order of $O\left(N^{2}\right)$. In the following, we will prove that the action profile specified in $\operatorname{BR} \_S C A\left(p, n_{i}, i\right)$ is a subgame perfect Nash equilibrium for the $N$ SUs' single-channel access game.

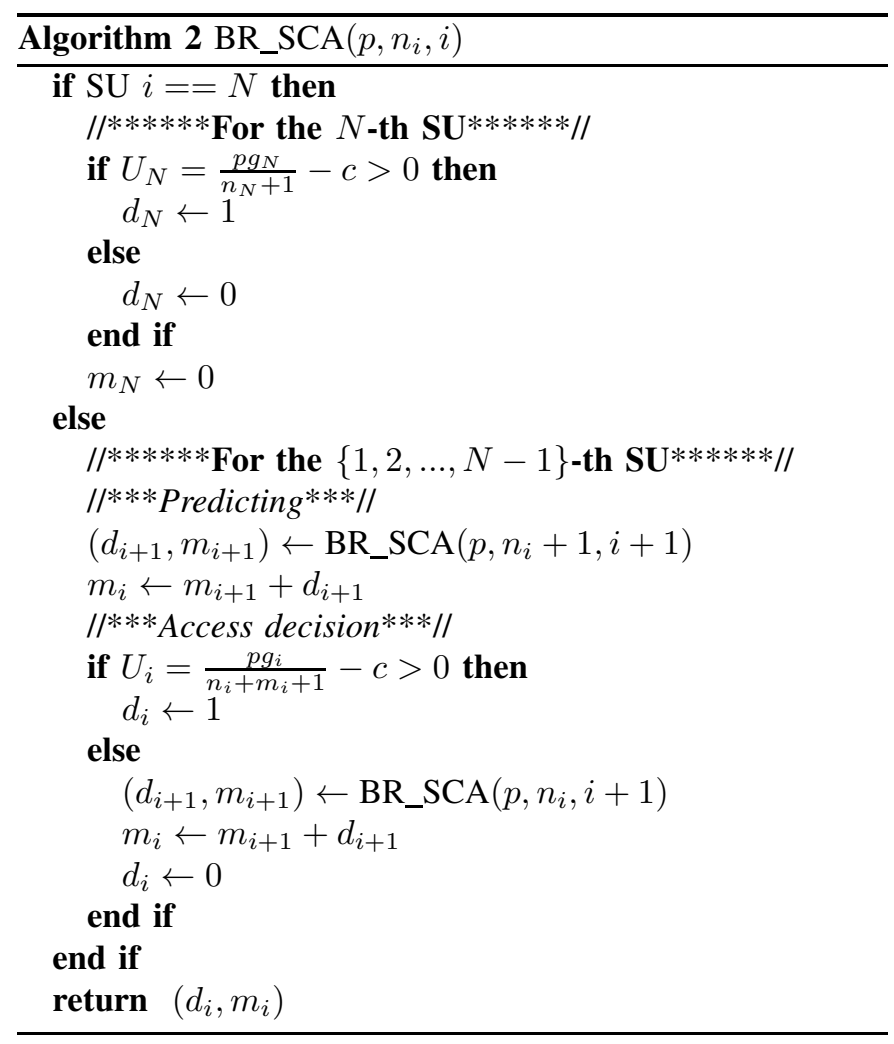

\section{B. Subgame Perfect Nash Equilibrium}

In this subsection, we will show that Algorithm 2 leads to the subgame perfect Nash equilibrium for the singlechannel access game. In the following, we first give the formal definitions of Nash equilibrium, subgame and subgame perfect Nash equilibrium as follows.

Definition 1: Given SUs' belief $p=\operatorname{Po}\left(\theta=\mathcal{H}_{0}\right)$, the action profile $\mathbf{d}^{*}=\left\{d_{1}^{*}, d_{2}^{*}, \ldots, d_{N}^{*}\right\}$ is a Nash equilibrium of the $N$ SUs' single-channel access game if and only if $\forall i \in\{1,2, \ldots, N\}, d_{i}^{*}=\operatorname{BR}_{i}\left(p, \sum_{\zeta \neq i} d_{\zeta}^{*}\right)$ as given in (27).

Definition 2: A subgame of the $N$ SUs' single-channel access game consists of the following three elements: 1) it starts from the $i$-th SU with $i=1,2, \ldots, N ; 2)$ it has the belief at current time slot, $p=\operatorname{Po}\left(\theta=\mathcal{H}_{0}\right)$; 3) it has current observation, $n_{i}$, which is the number of previous SUs who decide to access the channel.

Definition 3: A Nash equilibrium is a subgame perfect Nash equilibrium if and only if it is a Nash equilibrium for every subgame.

With the above definitions, we show in the following theorem that the action profile derived by Algorithm 2 is a subgame perfect Nash equilibrium of the single-channel access game.

Theorem 1: Given SUs' belief $p=\operatorname{Po}\left(\theta=\mathcal{H}_{0}\right)$, the action profile $\mathbf{d}^{*}=\left\{d_{1}^{*}, d_{2}^{*}, \ldots, d_{N}^{*}\right\}$, with $d_{i}^{*}$ being determined by BR_SCA $\left(p, n_{i}, i\right)$ and $n_{i}=\sum_{\zeta=1}^{i-1} d_{\zeta}^{*}$, is a subgame perfect Nash equilibrium for the $N$ SUs' single-channel access game.

Proof: We first show that $d_{\zeta}^{*}$ is the best response of the $\zeta$-th SU in the subgame starts from the $i$-th SU $(\forall 1 \leq i \leq$ $\zeta \leq N)$.

If $\zeta=N$, we can see that BR_SCA $\left(p, n_{N}, N\right)$ assigns the value of $d_{N}^{*}$ directly as

$$
d_{N}^{*}=\left\{\begin{array}{cc}
1, & \text { if } U_{N}=\frac{p g_{N}}{n_{N}+1}-c>0 ; \\
0, & \text { otherwise. }
\end{array}\right.
$$

Since $n_{N}=n_{-N}$, we have $d_{\zeta}^{*}=\operatorname{BR}_{\zeta}\left(p, n_{-\zeta}\right)$ in the case of $\zeta=N$ according to (27), i.e. $d_{\zeta}^{*}$ is the $\zeta$-th SU's best response.

If $\zeta<N$, suppose $d_{\zeta}^{*}$ is the $\zeta$-th SU's best response derived by BR_SCA $\left(p, n_{\zeta}, \zeta\right)$. If $d_{\zeta}^{*}=0$, denoting $d_{\zeta}^{\prime}=1$ as the contradiction, we can see from $\operatorname{BR} \_\operatorname{SCA}\left(p, n_{\zeta}, \zeta\right)$ that

$$
\left.U_{\zeta}\right|_{d_{\zeta}^{\prime}=1}=\frac{p g_{\zeta}}{n_{\zeta}+m_{\zeta}+1}-c<0=\left.U_{\zeta}\right|_{d_{\zeta}^{*}=0}
$$

which means that the $\zeta$-th SU has no incentive to deviate from $d_{\zeta}^{*}=1$ given the prediction of other SUs' decisions. If $d_{\zeta}^{*}=1$, denoting $d_{\zeta}^{\prime}=0$ as the contradiction, we can see from $\mathrm{BR} \_\mathrm{SCA}\left(p, n_{\zeta}, \zeta\right)$ that

$$
\left.U_{\zeta}\right|_{d_{\zeta}^{\prime}=0}=0<\left.U_{\zeta}\right|_{d_{\zeta}^{*}=1}=\frac{p g_{\zeta}}{n_{\zeta}+m_{\zeta}+1}-c,
$$

which means that the $\zeta$-th SU has no incentive to deviate from $d_{\zeta}^{*}=0$ given the prediction of other SUs' decisions. Therefore, $d_{\zeta}^{*}=\operatorname{BR} \_\operatorname{SCA}\left(p, n_{\zeta}, \zeta\right)$ is the $\zeta$-th SU's best response in the subgame of the single-channel access game starting with the $i$-th SU. Moreover, since the statement is true for $\forall \zeta$ satisfying $i \leq \zeta \leq N$, we know that $\left\{d_{i}^{*}, d_{i+1}^{*}, \ldots, d_{N}^{*}\right\}$ is the Nash equilibrium for the subgame starting from the $i$-th SU. Finally, according to the definition of subgame perfect Nash equilibrium, we can conclude that Theorem 1 is true.

\section{Homogeneous Case}

From the previous subsection, we know that a recursive procedure is needed to determine the best response profile of the $N$ SUs' single-channel access game. This is due to the fact that we need to predict the decisions of all subsequent SUs in order to determine the best response of a certain SU. In this subsection, we simplify the game with homogeneous setting to derive more concise best response.

In the homogeneous case, we assume that all SUs have the same form of expected utility function, i.e., $g_{i}=g, \forall i$. Under such a setting, the equilibrium can be characterized in a much simpler way.

Lemma 1: In the $N$ SUs' single channel access game under homogeneous settings, if $\mathbf{d}^{*}=\left\{d_{1}^{*}, d_{2}^{*}, \ldots, d_{N}^{*}\right\}$ is the Nash equilibrium action profile specified by BR_SCA () , then we have $d_{i}^{*}=1$ if and only if $0 \leq i \leq n^{*}$, where $n^{*}=\sum_{\zeta=1}^{N} d_{\zeta}^{*}$. 
Proof: Suppose the $i$-th SU's best response, $d_{i}^{*}=0$. Then, according to Algorithm 2, we have

$$
U_{i}=\frac{p g}{n_{i}+\left.m_{i}\right|_{d_{i}=1}+1}-c \leq 0 .
$$

The prediction of $m_{i}$ under the condition of $d_{i}=1$ relies on the recursive estimations of all subsequent SUs' channel access decisions. In particular, we have $\left.m_{i}\right|_{d_{i}=1}=\left.d_{i+1}\right|_{d_{i}=1}+$ $\left.m_{i+1}\right|_{d_{i}=1}$, where the value of $\left.d_{i+1}\right|_{d_{i}=1}$ can be computed as follows:

$$
\left.d_{i+1}\right|_{d_{i}=1}= \begin{cases}1, & \text { if }\left.\quad U_{i+1}\right|_{d_{i}=1}>0 \\ 0, & \text { otherwise }\end{cases}
$$

with

$$
\left.U_{i+1}\right|_{d_{i}=1}=\frac{p g}{n_{i}+1+\left.m_{i+1}\right|_{d_{i}=1}+1}-c .
$$

Since $1+\left.m_{i+1}\right|_{d_{i}=1} \geq\left. d_{i+1}\right|_{d_{i}=1}+\left.m_{i+1}\right|_{d_{i}=1}=\left.m_{i}\right|_{d_{i}=1}$, according to (36) and (38), we have

$$
\left.U_{i+1}\right|_{d_{i}=1} \leq U_{i} \leq 0,
$$

which means $\left.d_{i+1}\right|_{d_{i}=1}=0$. Following the same argument, we can show that $\left.d_{\zeta}\right|_{d_{i}=1}=0$ for all $\zeta=i+1, i+2, \ldots, N$. Therefore, we have

$$
\left.m_{i}\right|_{d_{i}=1}=\left.\sum_{\zeta=i+1}^{N} d_{\zeta}\right|_{d_{i}=1}=0 .
$$

Then, let us consider the $(i+1)$-th SU's best response, which can be calculated by

$$
d_{i+1}^{*}=\left\{\begin{array}{l}
1, \quad \text { if } U_{i+1}>0 \\
0, \quad \text { otherwise }
\end{array}\right.
$$

where

$$
U_{i+1}=\frac{p g}{n_{i+1}+\left.m_{i+1}\right|_{d_{i+1=1}}+1}-c .
$$

Since $n_{i+1}=n_{i}+d_{i},\left.m_{i}\right|_{d_{i}=1}=0$ and $\left.m_{i+1}\right|_{d_{i+1}=1} \geq 0$, we have $n_{i+1}+\left.m_{i+1}\right|_{d_{i+1}=1}+1 \geq n_{i}+\left.m_{i}\right|_{d_{i}=1}+1$. Comparing (36) and (42), we have $d_{i+1}^{*}=0$.

Following the same argument, we can show that if $d_{i}^{*}=0$, then $d_{\zeta}^{*}=0$ for all $\zeta \in\{i+1, i+2, \ldots, N\}$. Since all decisions can take values of either 0 or 1 , we have $d_{i}^{*}=1$ if and only if $0 \leq i \leq \sum_{\zeta=1}^{N} d_{\zeta}^{*}$. This completes the proof.

From Lemma 1, we can see that there exists a threshold structure in the Nash equilibrium profile of single-channel access game with homogeneous setting. The threshold structure is embodied in the fact that if $d_{i}^{*}=0$, then $d_{\zeta}^{*}=0, \forall \zeta \in$ $\{i+1, i+2, \ldots, N\}$, which means that as long as the $i$-th SU decides not to access the channel, then all subsequent SUs will not access the channel. On the other hand, if $d_{i}^{*}=1$, then $d_{\zeta}^{*}=1, \forall \zeta \in\{1,2, \ldots i-1\}$, which means that as long as the $i$-th SU decides to access the channel, then all previous SUs also access the channel. Moreover, according to the expected utility function definition in (5), the threshold can be illustrated by

$$
d_{i}^{*}=\left\{\begin{array}{l}
1, \quad \forall i<\left\lceil\frac{p g}{c}\right\rceil ; \\
0, \quad \forall i \geq\left\lceil\frac{p g}{c}\right\rceil .
\end{array},\right.
$$

where $\left\lceil\frac{p g}{c}\right\rceil$ can be interpreted as the maximal number of SUs that the channel can hold. This result can be easily extended to the multi-channel access game without resource constraint under the homogeneous setting as shown in the following theorem.

Theorem 2: In the $N$ SUs' multi-channel access game without resource constraint, if all the SUs have the same gain function $g_{i, j}=g_{j}$, there exists a threshold structure in the Nash equilibrium matrix $\mathbf{D}^{*}$ denoted by (4), i.e., for any row $j \in\{1,2, \ldots, M\}$ of $\mathbf{D}^{*}$, it holds

$$
d_{i, j}^{*}=\left\{\begin{array}{c}
1, \quad \forall i<\left\lceil\frac{p_{j} g_{j}}{c_{j}}\right\rceil ; \\
0, \quad \forall i \geq\left\lceil\frac{p_{j} g_{j}}{c_{j}}\right\rceil .
\end{array}\right.
$$

Proof: This theorem directly follows by extending Lemma 1 into $M$ independent channels case.

\section{Multi-Channel Access Game with Resource CONSTRAINT}

In this section, we study the multi-channel access game with resource constraint, which is corresponding to the case with $L<M$ in (7). Unlike previous case, when there is resource constraint for each SU, the different channel accesses are no longer independent but coupled with each other instead. In the following, we will discuss a recursive algorithm that can characterize the subgame perfect Nash equilibrium of the multi-channel access game with resource constraint.

\section{A. Recursive Best Response Algorithm}

In the resource constraint case, we assume that each SU can at most access $L$ channels at each time slot with $L<M$. In such a case, the $i$-th SU's best channels access decision can be found by the following optimization problem.

$$
\begin{gathered}
\mathbf{d}_{i}^{*}=\mathrm{BR}_{i}\left(\mathbf{p}, \mathbf{n}_{-i}\right)=\underset{\mathbf{d}_{i} \in\{0,1\}^{M}}{\arg \max } \sum_{j=1}^{M} d_{i, j} \cdot U_{i, j}, \\
\text { s.t. } \sum_{j=1}^{M} d_{i, j} \leq L<M,
\end{gathered}
$$

where $\mathbf{p}=\left\{p_{1}, p_{2}, \ldots, p_{M}\right\}$ is SUs' beliefs on the states of all primary channels and $U_{i, j}$ is given in (5). From (45), we can see that the $i$-th SU's decision on whether to access channel $\mathrm{Ch}_{j}$ is coupled with the decisions on all other channels, and thus (45) cannot be decomposed into $M$ subproblems. Nevertheless, we can still find the best response of each SU by comparing all possible combinations of $L$ channels. Let $\boldsymbol{\Phi}=\left\{\phi_{1}, \phi_{2}, \ldots, \phi_{H}\right\}$ denote the set of all combinations of $l(1 \leq l \leq L)$ channels out of $M$ channels, where $H=\sum_{l=1}^{L} \mathrm{C}_{M}^{l}=\sum_{l=1}^{L} \frac{M !}{l !(M-l) !}$ and $\phi_{h}=\left(\phi_{h, 1}, \phi_{h, 2}, \ldots, \phi_{h, M}\right)^{\prime}$ is one possible combination with $\phi_{h, j}$ representing whether channel $\mathrm{Ch}_{j}$ is selected to access to, e.g.,

$$
\phi_{h}=(\underbrace{1,1, \ldots, 1}_{l}, \underbrace{0,0, \ldots, 0}_{M-l})^{\prime}
$$

means the $\mathrm{SU}$ accesses channel $\mathrm{Ch}_{1}, \mathrm{Ch}_{2}, \ldots, \mathrm{Ch}_{l}(1 \leq l \leq L)$. In other words, $\boldsymbol{\Phi}$ is the candidate strategy set of each SU with resource constraint $L$. 
Let us define the $i$-th SU's observation of previous SUs' channel access decisions as

$$
\mathbf{n}_{i}=\left\{n_{i, 1}, n_{i, 2}, \ldots, n_{i, M}\right\}
$$

where $n_{i, j}=\sum_{k=1}^{i-1} d_{k, j}$ is the number of SUs deciding to access channel $\mathrm{Ch}_{j}$ before the $i$-th SU. Let $\mathbf{m}_{i}$ denote the subsequent SUs' decisions after the $i$-th $\mathrm{SU}$, we have its recursive form as

$$
\mathbf{m}_{i}=\mathbf{m}_{i+1}+\mathbf{d}_{i+1} .
$$

Then, let

$$
\left.\mathbf{m}_{i}\right|_{\mathbf{d}_{i}=\phi_{h}}=\left\{\left.m_{i, 1}\right|_{\mathbf{d}_{i}=\phi_{h}},\left.m_{i, 2}\right|_{\mathbf{d}_{i}=\phi_{h}}, \ldots,\left.m_{i, M}\right|_{\mathbf{d}_{i}=\phi_{h}}\right\}
$$

with $\left.m_{i, j}\right|_{\mathbf{d}_{i}=\phi_{h}}$ being the predicted number of subsequent SUs who will access channel $\mathrm{Ch}_{j}$ under the condition of $\mathbf{d}_{i}=$ $\phi_{h}$, where $\mathbf{d}_{i}=\left(d_{i, 1}, d_{i, 1}, \ldots, d_{i, M}\right)^{\prime}$ and $\phi_{h} \in \mathbf{\Phi}$. In such a case, the predicted number of SUs accessing each primary channel excluding the $i$-th $\mathrm{SU}$ is

$$
\left.\hat{\mathbf{n}}_{-i}\right|_{\mathbf{d}_{i}=\phi_{h}}=\mathbf{n}_{i}+\left.\mathbf{m}_{i}\right|_{\mathbf{d}_{i}=\phi_{h}} .
$$

According to above definitions, we can write the $i$-th SU's expected utility by accessing channel $\mathrm{Ch}_{j}$ when $\mathbf{d}_{i}=\phi_{h}$ as

$$
\left.U_{i, j}\right|_{\mathbf{d}_{i}=\phi_{h}}=\frac{p_{j} g_{j}}{n_{i, j}+\left.m_{i, j}\right|_{\mathbf{d}_{i}=\phi_{h}}+\phi_{h, j}}-c_{j}
$$

Then, the total expected utility the $i$-th SU can obtain with $\mathbf{d}_{i}=\phi_{h}$ is the sum of $\left.U_{i, j}\right|_{\mathbf{d}_{i}=\phi_{h}}$ over all $M$ channels, i.e.,

$$
\left.U_{i}\right|_{\mathbf{d}_{i}=\phi_{h}}=\left.\sum_{j=1}^{M} U_{i, j}\right|_{\mathbf{d}_{i}=\phi_{h}} .
$$

In such a case, we can find the optimal $\phi_{h}^{*}$ which maximizes the $i$-th SU's expected utility $\left.U_{i}\right|_{\mathbf{d}_{i}=\phi_{h}}$ as follows

$$
\phi_{h}^{*}=\underset{\phi_{h} \in \Phi}{\arg \max }\left\{\left.U_{i}\right|_{\mathbf{d}_{i}=\phi_{h}}\right\} .
$$

To obtain the best response in (53), each SU needs to calculate the expected utilities defined in (51), which in turn requires to predict $\left.m_{i, j}\right|_{\mathbf{d}_{i}=\phi_{h}}$, i.e., the number of SUs who access channel $\mathrm{Ch}_{j}$ after the $i$-th SU. For the $N$-th SU who has already known all previous SUs' channel access decisions, no prediction is required. Therefore, similar to Algorithm 2, given belief $\mathbf{p}=\left\{p_{1}, p_{2}, \ldots, p_{M}\right\}$ at current time slot and current observation $\mathbf{n}_{i}=\left\{n_{i, 1}, n_{i, 2}, \ldots, n_{i, M}\right\}$, we design another recursive best response algorithm BR_MCA $\left(\mathbf{p}, \mathbf{n}_{i}, i\right)^{3}$ for solving the multi-channel access game with resource constraint in Algorithm 3. As we can see, the $N$-th SU only needs to compare the expected utilities of accessing all $M$ channels respectively and choose $L$ or less than $L$ channels with highest positive expected utilities. Note that $\max ^{L}$ means finding the highest $L$ values. For other SUs, each one needs to first recursively predict the subsequent SUs' decisions, and then make his/her own decision based on the prediction and current observations. We can see that the complexity of Algorithm 3 is determined by both the number of SUs $N$ and the number of primary channels $M$. Since each SU needs to run all the possible combinations $\phi_{h}$ and each combination

${ }^{3} \mathrm{MCA}$ is abbreviation for multi-channel access. complexity is polynomial with the number of primary channels $M$, the total complexity of Algorithm 3 is at the order of $O\left(H N^{2}\right)$, where $H=\sum_{l=1}^{L} \frac{M !}{l !(M-l) !}$ is at the polynomial order of $M$.

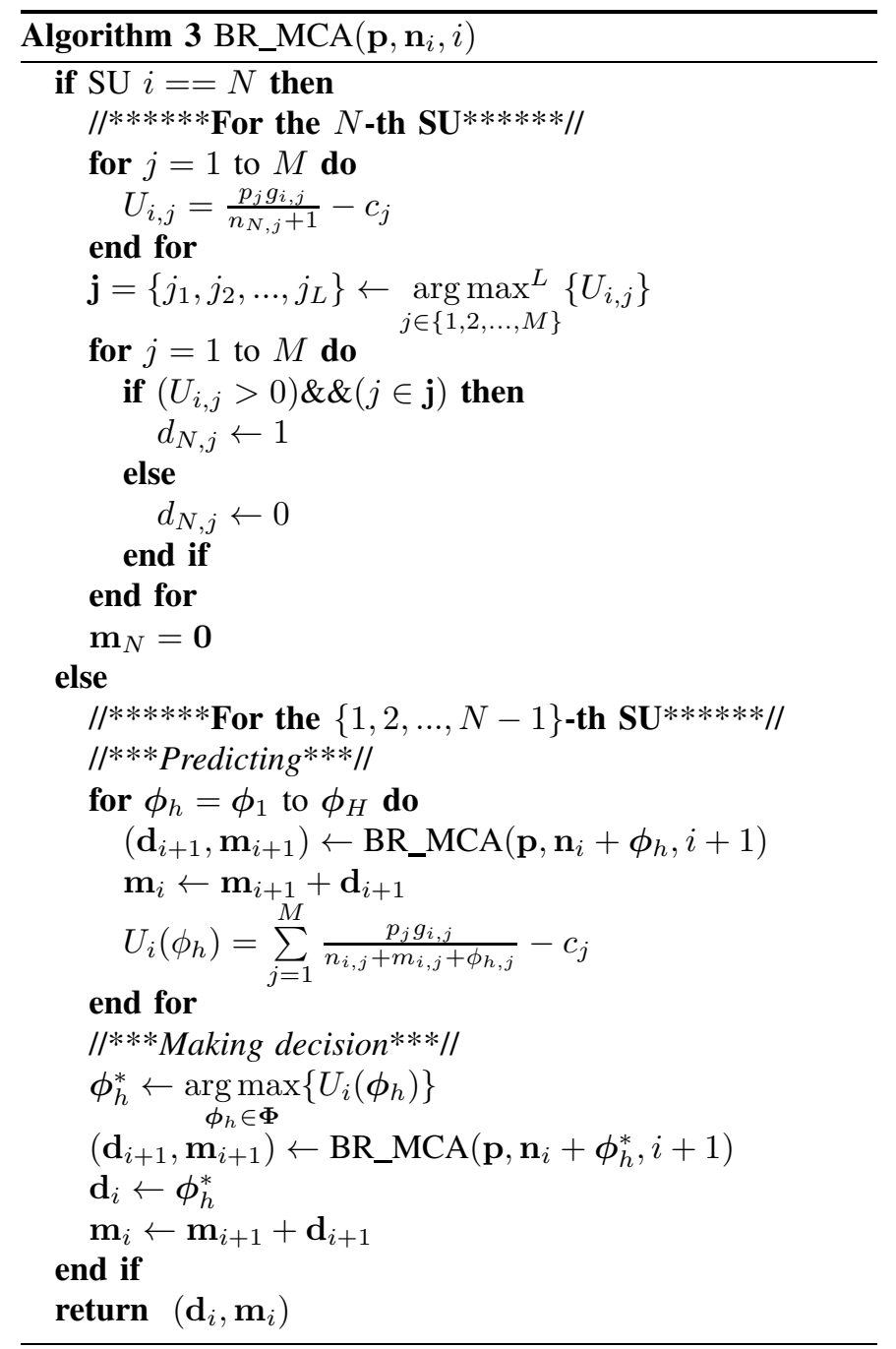

\section{B. Subgame Perfect Nash Equilibrium}

Similar to the single-channel access game, we first give formal definitions of the Nash equilibrium and subgame of multi-channel access game with resource constraint.

Definition 4: Given SUs' belief $\mathbf{p}=\left\{p_{1}, p_{2}, \ldots, p_{M}\right\}$ with $p_{j}=\operatorname{Po}\left(\theta_{j}=\mathcal{H}_{0}\right)$, the action profile $\mathbf{D}^{*}=\left\{\mathbf{d}_{1}^{*}, \mathbf{d}_{2}^{*}, \ldots, \mathbf{d}_{N}^{*}\right\}$ is a Nash equilibrium of the $N$ SUs' multi-channel access game with resource constraint $L$, if and only if $\mathbf{d}_{i}^{*}=$ $\mathrm{BR}_{i}\left(\mathbf{p}, \sum_{\zeta \neq i} \mathbf{d}_{\zeta}^{*}\right)$ as defined in (45) for all $i$.

Definition 5: A subgame of the $N$ SU's multi-channel access game with resource constraint $L$ consists of the following three elements: 1) it starts from the $i$-th $\mathrm{SU}$ with $i=1,2, \ldots, N ; 2)$ it has the belief at current time slot, $\mathbf{p}=\left\{p_{1}, p_{2}, \ldots, p_{M}\right\}$ with $\left.p_{j}=\operatorname{Po}\left(\theta_{j}=\mathcal{H}_{0}\right) ; 3\right)$ it has current observation, $\mathbf{n}_{i}$, which is the number of previous SUs who decide to access the channel. 
Based on Definition 3, 4 and 5, we show in the following theorem that the action profile obtained by Algorithm 3 is a subgame perfect Nash equilibrium of the multi-channel access game with resource constraint.

Theorem 3: Given SUs' belief $\mathbf{p}=\left\{p_{1}, p_{2}, \ldots, p_{M}\right\}$ with $p_{j}=\operatorname{Po}\left(\theta_{j}=\mathcal{H}_{0}\right)$, the action profile $\mathbf{D}^{*}=\left\{\mathbf{d}_{1}^{*}, \mathbf{d}_{2}^{*}, \ldots, \mathbf{d}_{N}^{*}\right\}$, where $\mathbf{d}_{i}^{*}$ determined by BR_MCA $\left(\mathbf{p}, \mathbf{n}_{i}, i\right)$ and $\mathbf{n}_{i}=\sum_{\zeta=1}^{i-1} \mathbf{d}_{\zeta}^{*}$, is a subgame perfect Nash equilibrium for the $N$ SUs' multichannel access game.

Proof: The proof of this theorem is similar to that of Theorem 1, the details of which are omitted due to page limitation. The proof outline is to first show $\forall i, \zeta, \mathbf{d}_{\zeta}^{*}$ is the best response of the $\zeta$-th $\mathrm{SU}$ in the subgame starting from the $i$-th SU by analyzing two cases: $\zeta=N$ and $\zeta<N$. Then, we can show that $\left\{\mathbf{d}_{i}^{*}, \mathbf{d}_{i+1}^{*}, \ldots, \mathbf{d}_{N}^{*}\right\}$ is the Nash equilibrium for the subgame starting from the $i$-th SU. Finally, according to the definition of subgame perfect Nash equilibrium, we can conclude that Theorem 3 is true.

\section{Simulation Results}

In this section, we conduct simulation to verify the performance of the proposed cooperative channel learning method and recursive best response algorithms. We simulate a primary network with five independent primary channels $\left\{\mathrm{Ch}_{1}, \mathrm{Ch}_{2}, \mathrm{Ch}_{3}, \mathrm{Ch}_{4}, \mathrm{Ch}_{5}\right\}$. The utilization ratios set is assumed to be finite as $\boldsymbol{\Lambda}=\left\{\Lambda_{1}=0.1, \Lambda_{2}=0.3, \Lambda_{3}=\right.$ $\left.0.5, \Lambda_{4}=0.7, \Lambda_{5}=0.9\right\}$. SUs' detection probability and false alarm probability are set as $P_{d}=0.9$ and $P_{f}=0.1$, respectively, which mean that after perform spectrum sensing, each SU can observe a signal following conditional distribution of $f\left(s_{+} \mid \theta=\mathcal{H}_{1}\right)=0.9$ and $f\left(s_{+} \mid \theta=\mathcal{H}_{0}\right)=0.1$.

\section{A. Cooperative Channel Learning}

In this subsection, we evaluate the performance of the proposed cooperative channel learning method, including the utilization ratio learning and channel state learning. In the simulation, we set the number of SUs as $N=10$ and the utilization ratio of each primary channel as $\left\{\lambda_{1}=\Lambda_{1}, \lambda_{2}=\right.$ $\left.\Lambda_{2}, \lambda_{3}=\Lambda_{3}, \lambda_{4}=\Lambda_{4}, \lambda_{5}=\Lambda_{5}\right\}$. In such a case, a correct estimation of the utilization ratio means that

$$
P^{*}\left(\lambda_{j}=\Lambda_{j}\right)=1, P^{*}\left(\lambda_{j} \neq \Lambda_{j}\right)=0, \forall j .
$$

At the beginning of the simulation, we assign SUs' initial estimation of utilization ratio as $P^{(0)}\left(\lambda_{j}=\Lambda_{k}\right)=0.2, \forall j, \Lambda_{k}$. At every time slot, after performing spectrum sensing, SUs exchange their observed signals and cooperatively update the estimation of the utilization ratio using learning rule in (18). Fig. 3 shows the channel utilization ratio learning curves of four cases: SUs at most sense $L=\{2,3,4,5\}$ channels. The $\mathrm{x}$-axis is the simulation time, and the $\mathrm{y}$-axis is the difference between SU's estimation of utilization ratio at each time slot, $P^{(t)}$, and the true utilization ratio $P^{*}$, which can be calculated by

$$
\operatorname{Diff}^{(t)}=\frac{1}{5} \sum_{j=1}^{5} \sum_{k=1}^{5}\left[P^{(t)}\left(\lambda_{j}=\Lambda_{k}\right)-P^{*}\left(\lambda_{j}=\Lambda_{k}\right)\right]^{2} .
$$

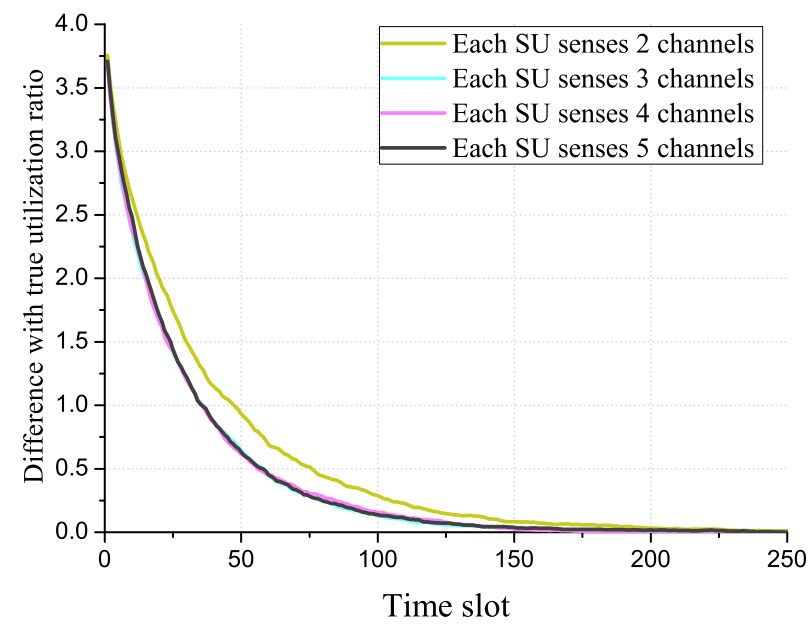

Fig. 3. Channel utilization ratio learning performance.

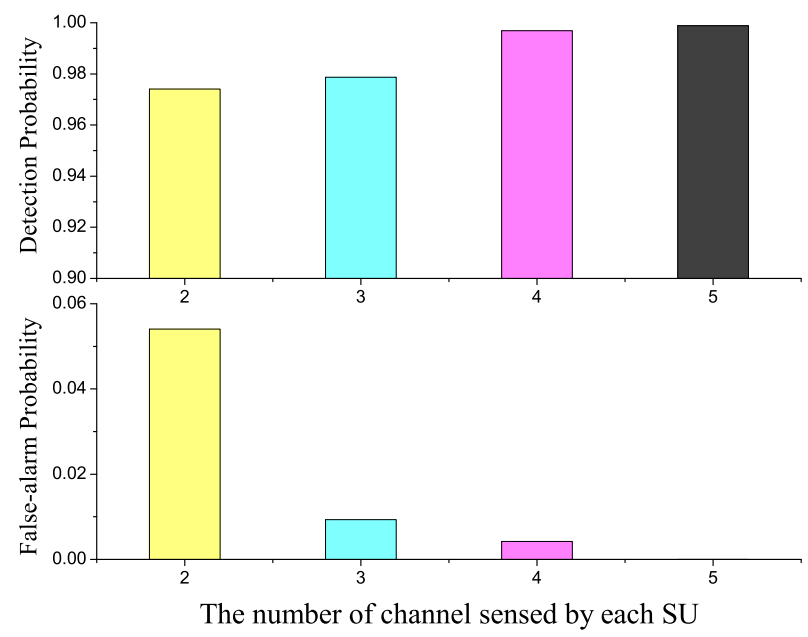

Fig. 4. Detection and false-alarm probabilities.

From the figure, we can see that the difference goes to zero finally for all four cases, i.e., SUs can effectively learn the true utilization ratio of each channel. Moreover, the case that each SU can only sense 2 channels converges slower than other cases, which is because fewer signals are observed by each SU at every time slot when the resource constraint is $L=2$.

At every time slot, after channel utilization ratio learning, SUs also need to learn the current channel state based on the observed signals and estimated channel utilization ratio. Fig. 4 shows the detection probability and false-alarm probability of four cases $L=\{2,3,4,5\}$, when SUs adopting our proposed channel state learning rule in (22). For all four cases, the detection probabilities are enhanced compared with single SU's $P_{d}=0.9$ and the false-alarm probabilities decrease compared with single SU's $P_{f}=0.1$. Specifically, when each SU can sense 5 channels at every time slot, the detection probability goes to 1 and false-alarm probability is nearly 0 . Moreover, similar to the performance of channel utilization ratio learning, the case that each SU can only sense 2 channels performs the worst due to the limited observed signals. 
TABLE I

NASH EQUILIBRIUM MATRIX $\mathbf{D}^{*}$

\begin{tabular}{l|llllllllll} 
& 1 & 2 & 3 & 4 & 5 & 6 & 7 & 8 & 9 & 10 \\
\hline $\mathrm{Ch}_{1}$ & 1 & 1 & 1 & 1 & 1 & 1 & 1 & 1 & 1 & 0 \\
$\mathrm{Ch}_{2}$ & 1 & 1 & 1 & 1 & 0 & 0 & 0 & 0 & 0 & 0 \\
$\mathrm{Ch}_{3}$ & 1 & 1 & 1 & 0 & 0 & 0 & 0 & 0 & 0 & 0 \\
$\mathrm{Ch}_{4}$ & 1 & 1 & 0 & 0 & 0 & 0 & 0 & 0 & 0 & 0 \\
$\mathrm{Ch}_{5}$ & 1 & 0 & 0 & 0 & 0 & 0 & 0 & 0 & 0 & 0
\end{tabular}

\section{B. Multi-Channel Access Game without Resource Constraint}

In this subsection, we evaluate the performance of the proposed best response algorithm for multi-channel access game without resource constraint. We first simulate the homogenous case to verify the threshold property of the Nash equilibrium matrix, i.e., Theorem 2, and the influence of different decision making orders on SUs' utilities, i.e., making decisions earlier may have advantage. Then, we compare the performance of the proposed best response algorithm, i.e., Algorithm 2, with the performances of other algorithms under heterogenous settings.

For the homogenous case, we set all SUs' gain function as $g_{i, j}=1$. At each time slot, we let SUs sequentially make decisions based on their estimated state of each channel according to Algorithm 2. In the first simulation, we set the SU number as $N=10$ to specifically verify the threshold structure of Nash equilibrium matrix. Moreover, in order to verify different threshold structures for different channel parameters as illustrated in Theorem 2, we set the costs of accessing five primary channels as $\left\{c_{1}=0.1, c_{2}=0.2, c_{3}=\right.$ $\left.0.3, c_{4}=0.4, c_{5}=0.5\right\}$, respectively. Under such settings, we can see that when all primary channels are in idle state, the access thresholds for five channels should be $\{9,4,3,2,1\}$ according to Theorem 2, e.g., the threshold 9 means only the $(1 \sim 9)$-th SUs should access channel $\mathrm{Ch}_{1}$. Table I shows the Nash equilibrium matrix $\mathbf{D}^{*}$ derived by Algorithm 2 when all primary channels are in idle state, where each column contains one SU's decisions $\left\{d_{i, j}, \forall j\right\}$ and each row contains all SUs' access decisions on one specific channel $\mathrm{Ch}_{j}$, i.e., $\left\{d_{i, j}, \forall i\right\}$. From Table I, we can see that once a SU does not access some specific channels, all the subsequent SUs will not access that channel, which is consistent with the conclusion in Theorem 2. Moreover, the access thresholds of all five channels exactly match the theoretical value $\{9,4,3,2,1\}$, which further verifies the correctness of Theorem 2.

From Table I, we can further see that SUs who make decisions earlier have advantage, e.g., the first SU can access all channels while the last SU fails to access any channel. Therefore, in the second simulation of the homogenous case, we dynamically adjust the order of decision making to ensure the fairness, and set the SU number as $N=5$ to specifically show each SU's utility. In Fig. 5, we show all SUs' utilities along with the simulation time, where the order of decision making changes every 100 time slots. In the first 100 time slots, during which the order of decision making is $1 \rightarrow 2 \rightarrow 3 \rightarrow 4 \rightarrow 5$, we can see that the first SU obtains the highest utility while the last SU obtains the lowest

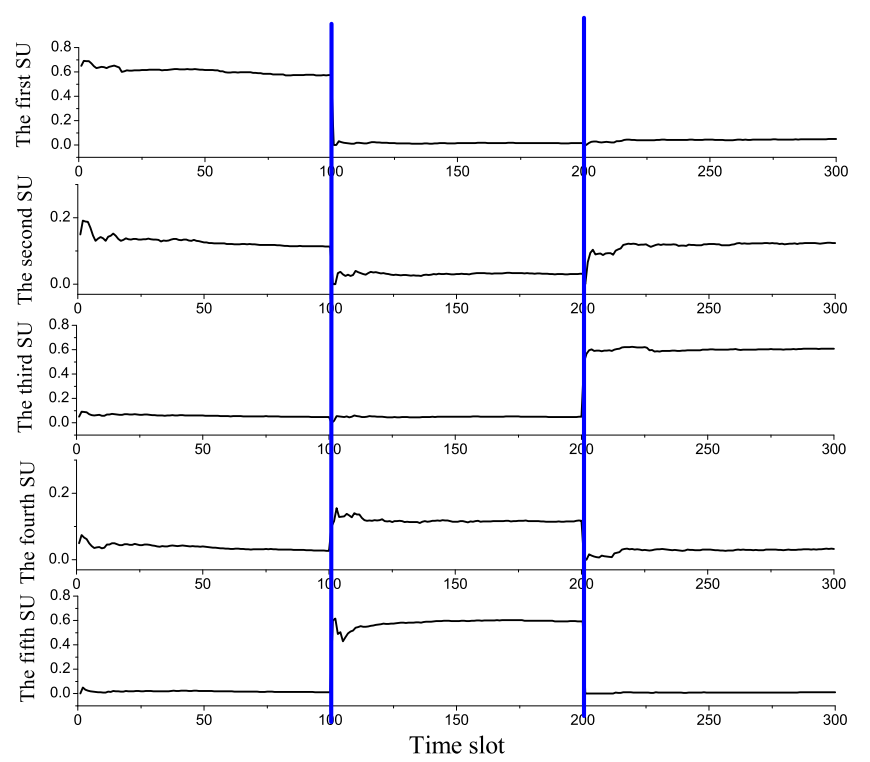

Fig. 5. Each SU's utility in homogenous case without resource constraint.

utility since he/she can only access 1 primary channel. In the second 100 time slots, we reverse the decision making order as $5 \rightarrow 4 \rightarrow 3 \rightarrow 2 \rightarrow 1$, which leads to that the first SU receives the lowest utility. Therefore, by periodically changing the order of decision making, we can expect that the utilities of all SUs will tend to be the same after a period of time. Note that in the heterogenous case, by putting the SU who has higher gain coefficient $g_{i, j}$ at the lower order can increase the social welfare. In such a case, the order that can maximize the social welfare will be the descending order of the SUs' gain coefficients $g_{i, j}$.

For the heterogenous case, we randomize each SU's gain function $g_{i, j}$ between 0 and 1 and set their prior belief as $\left\{p_{j}^{(0)}=0.5, \forall j\right\}$. In this simulation, we compare the performance in terms of SUs' social welfare, which is defined as the total utilities of all SUs, among different kinds of algorithms listed as follows:

- Best Response: The proposed recursive best response algorithm in Algorithm 2 with cooperative channel state learning.

- Myopic: At each time slot, the $i$-th SU selects channels only according to his/her current observation $\mathbf{n}_{i}=$ $\left\{n_{i, j}, \forall j\right\}$ without channel learning.

- Learning: At each time slot, each SU selects channels only according to the belief $p_{j}^{(t)}$ updated by cooperative channel learning without considering the negative network externality.

- Random: Each SU randomly accesses channels.

For the myopic and learning strategies, the $i$-th SU's expected utility of accessing channel $\mathrm{Ch}_{j}$ can be calculated by

$$
\begin{aligned}
U_{i, j}^{\mathrm{m}} & =p_{j}^{(0)} \frac{g_{i, j}}{n_{i, j}+d_{i, j}}-c_{j}, \\
U_{i, j}^{1} & =p_{j}^{(t)} \frac{g_{i, j}}{d_{i, j}}-c_{j} .
\end{aligned}
$$

With these expected utilities, both myopic and learning algorithm can be derived by (24). We can see that the myopic 


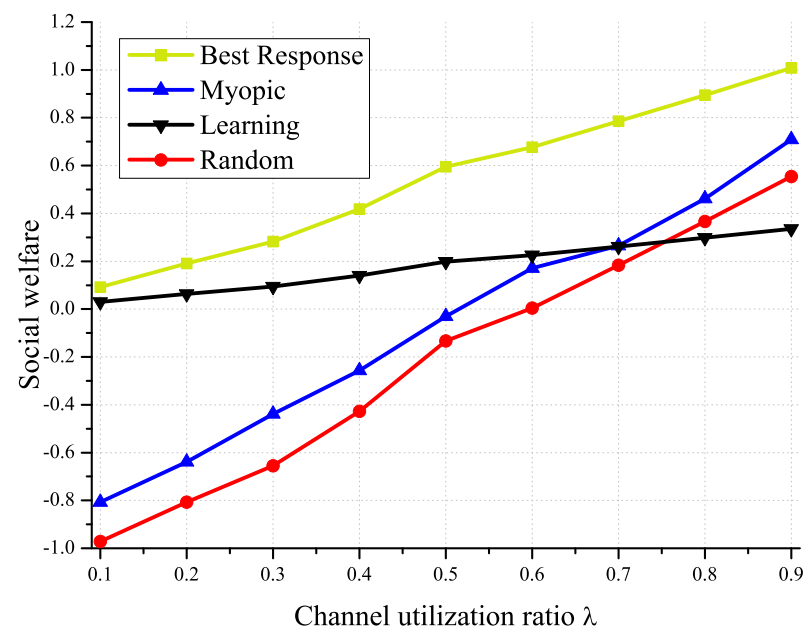

Fig. 6. Social welfare comparison without resource constraint.

strategy is without social learning and the learning strategy is without consideration of negative network externality. In the simulation, in order to verify the influence of channel utilization ratio on SUs' social welfare, we set the utilization ratios of all five primary channels as the same and adjust $\lambda$ from 0.1 to 0.9 , i.e., from very busy primary channel to very idle primary channel.

Fig. 6 shows the performance comparison result, where the $\mathrm{x}$-axis is the channel utilization ratio $\lambda$ and $\mathrm{y}$-axis is the social welfare averaged over hundred of time slots. From the figure, we can see with the increase of $\lambda$, the social welfare keeps increasing for all algorithms and our best response algorithm performs the best. When $\lambda \leq 0.7$, the learning algorithm performs better than myopic and random algorithms. This is because, when PUs occupy the channel with a relatively high probability, by adopting learning algorithm, although SUs do not consider the negative network externality, they can accurately estimate the channel state of each time slot and avoid to access the time slots when PUs are active. Moreover, when $\lambda \geq 0.8$, we can see that the learning algorithm performs the worst, which is because when the primary channels are very idle, considering other SUs' decisions, i.e., negative network externality, plays a more important role than channel state learning.

\section{Multi-Channel Access Game with Resource Constraint}

In this subsection, we evaluate the performance of the proposed best response algorithm for multi-channel access game with resource constraint $L=3$. Similar to the heterogenous case of the without-resource-constraint scenario, we randomize each SU's gain $g_{i, j}$ within $[0,1]$ and compare the performance of our proposed best response algorithm, i.e., Algorithm 3, with myopic, learning and random algorithms in terms of SUs' social welfare. For the myopic, learning and random algorithms, same resource constraint is adopted, i.e., each SU can at most access 3 channels. Fig. 7 shows the performance comparison result, from which we can see the phenomenon is similar to the case without resource constraint where our best response algorithm performs the best.

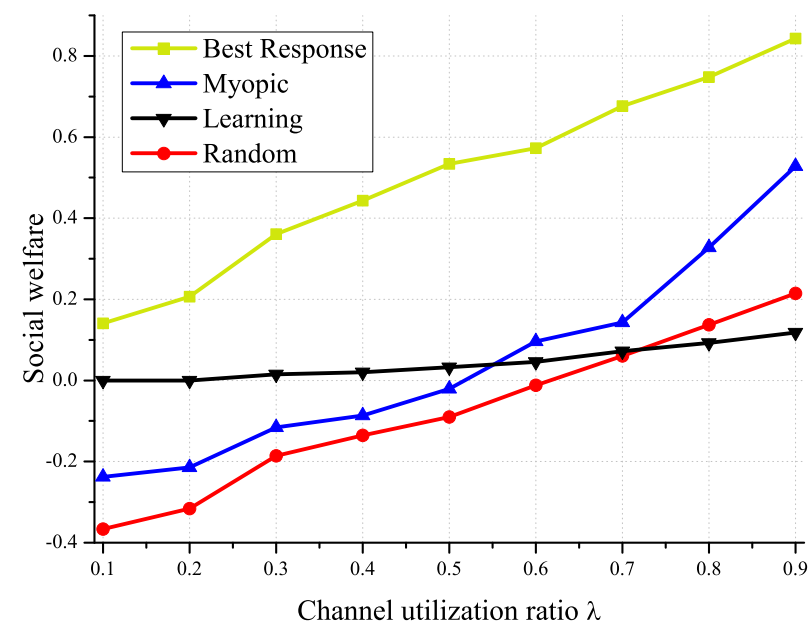

Fig. 7. Social welfare comparison with resource constraint.

\section{CONCLUSION}

In this paper, we proposed and studied the multiple SUs' multi-channel sensing and access game. For the multi-channel sensing problem, we designed a cooperative channel state learning method based on Bayesian learning rule. For the multi-channel access problem, we analyzed two scenarios: without resource constraint and with resource constraint, respectively, and designed two recursive best response algorithms for both scenarios to find the subgame perfect Nash equilibrium. Moreover, we also discussed the homogenous case of the scenario without resource constraint. Simulation results show that our proposed channel learning rule not only can effectively help SUs learn the channel utilization ratio, but also can enhance SUs' detection probability and decrease their false-alarm probability. Moreover, our proposed best response algorithms outperform myopic, learning and random algorithms in terms of social welfare.

\section{REFERENCES}

[1] Y.-C. Liang, K.-C. Chen, G. Y. Li, and P. Mahonen, "Cognitive radio networking and communications: an overview," IEEE Trans. Veh. Technol., vol. 60, no. 7, pp. 3386-3407, 2011.

[2] B. Wang and K. J. R. Liu, "Advances in cognitive radios: a survey," IEEE J. Sel. Topics Signal Process., vol. 5, no. 1, pp. 5-23, 2011.

[3] K. J. R. Liu and B. Wang, Cognitive Radio Networking and Security: A Game Theoretical View. Cambridge University Press, 2010.

[4] S. Haykin, "Cognitive radio: brain-empowered wireless communications," IEEE J. Sel. Areas Commun., vol. 23, no. 2, pp. 201-220, 2005.

[5] B. Wang, Y. Wu, and K. J. R. Liu, "Game theory for cognitive radio networks: an overview," Comput. Netw., vol. 54, no. 14, pp. 2537-2561, 2010.

[6] S. Shankar, C. Cordeiro, and K. Challapali, "Spectrum agile radios: utilization and sensing architectures," in Proc. 2005 IEEE DySPAN, pp. $160-169$.

[7] H. Tang, "Some physical layer issues of wide-band cognitive radio systems," in Proc. 2005 IEEE DySPAN, pp. 151-159.

[8] I. F. Akyildiz, B. F. Lo, and R. Balakrishnan, "Cooperative spectrum sensing in cognitive radio networks: a survey," Physical Commun., vol. 4, no. 3, pp. 40-62, 2011.

[9] R. Fan and H. Jiang, "Optimal multi-channel cooperative sensing in cognitive radio networks," IEEE Trans. Wireless Commun., vol. 9, no. 3, pp. 1128-1138, 2010 .

[10] Q. Zhao, L. Tong, A. Swami, and Y. Chen, "Decentralized cognitive MAC for opportunistic spectrum access in ad hoc networks: a POMDP framework," IEEE J. Sel. Areas Commun., vol. 25, no. 3, pp. 589-600, 2007. 
[11] J. Unnikrishnan and V. V. Veeravalli, "Algorithms for dynamic spectrum access with learning for cognitive radio," IEEE Trans. Signal Process., vol. 58, no. 2, pp. 750-760, 2010.

[12] A. K. Sadek, K. J. R. Liu, and A. Ephremides, "Cognitive multiple access via cooperation: protocol design and stability analysis," IEEE Trans. Inf. Theory, vol. 53, no. 10, pp. 3677-3696, 2007.

[13] A. A. El-Sherif, A. Kwasinski, A. Sadek, and K. J. R. Liu, "Contentaware cognitive multiple access protocol for cooperative packet speech communications," IEEE Trans. Wireless Commun., vol. 8, no. 2, pp. 995-1005, 2009.

[14] A. A. El-Sherif, A. K. Sadek, and K. J. R. Liu, "Opportunistic multiple access for cognitive radio networks," IEEE J. Sel. Areas Commun., vol. 29, no. 4, pp. 704-715, 2011.

[15] Y. Wu, B. Wang, K. J. R. Liu, and T. C. Clancy, "A scalable collusionresistant multi-winner cognitive spectrum auction game," IEEE Trans. Wireless Commun., vol. 57, no. 12, pp. 3805-3816, 2009.

[16] Z. Ji and K. J. R. Liu, "Dynamic spectrum sharing: a game theoretical overview," IEEE Commun. Mag., vol. 45, no. 5, pp. 88-94, 2010.

[17] D. Li, Y. Xu, X. Wang, and M. Guizani, "Coalitional game theoretic approach for secondary spectrum access in cooperative cognitive radio networks," IEEE Trans. Wireless Commun., vol. 10, no. 3, pp. 844-856, 2011.

[18] Y.-C. Liang, Y. Zeng, E. C. Peh, and A. T. Hoang, "Sensing-throughput tradeoff for cognitive radio networks," IEEE Trans. Wireless Commun., vol. 7, no. 4, pp. 1326-1337, 2008.

[19] A. A. El-Sherif and K. J. R. Liu, "Joint design of spectrum sensing and channel access in cognitive radio networks," IEEE Trans. Wireless Commun., vol. 10, no. 6, pp. 1743-1753, 2011.

[20] C. Jiang, Y. Chen, Y. Gao, and K. J. R. Liu, "Joint spectrum sensing and access evolutionary game in cognitive radio networks," IEEE Trans. Wireless Commun., vol. 12, no. 5, pp. 2470-2483, 2013.

[21] W. H. Sandholm, "Negative externalities and evolutionary implementation," Rev. Economic Studies, vol. 72, no. 3, pp. 885-915, 2005.

[22] C.-Y. Wang, Y. Chen, and K. J. R. Liu., "Chinese restaurant game," IEEE Signal Process. Lett., vol. 19, no. 12, pp. 898-901, 2012.

[23] C.-Y. Wang, Y. Chen, and K. J. R. Liu, "Sequential chinese restaurant game," IEEE Trans. Signal Process., vol. 61, no. 3, pp. 571-584, 2013.

[24] B. Zhang, Y. Chen, C.-Y. Wang, and K. J. R. Liu, "Learning and decision making with negative externality for opportunistic spectrum access," in Proc. 2012 IEEE Globecom, pp. 1404-1409.

[25] C. Jiang, Y. Chen, Y.-H. Yang, C.-Y. Wang, and K. J. R. Liu, "Dynamic Chinese restaurant game in cognitive radio networks," in Proc. 2013 IEEE INFOCOM, pp. 1-9.

[26] C. Jiang, Y. Chen, Y. Gao, and K. J. R. Liu, "Indian buffet game with non-bayesian social learning," in Proc. 2013 IEEE Globesip.

[27] — "Indian buffet game with negative network externality and nonbayesian social learning." Available: http://arxiv.org/abs/1309.2922.

[28] I. F. Akyildiz, W.-Y. Lee, M. C. Vuran, and S. Mohanty, "Next generation/dynamic spectrum access/cognitive radio wireless networks: a survey," Comput. Netw., vol. 50, no. 9, pp. 2127-2159, 2006.

[29] D. Gale and S. Kariv, "Bayesian learning in social networks," Games Economic Behavior, vol. 45, no. 11, pp. 329-346, 2003.

[30] R. Swinburne, Bayes's Theorem. Oxford University Press, 2002.

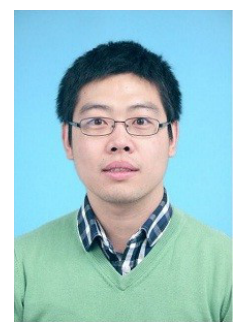

Chunxiao Jiang (S'09-M'13) received his B.S. degree in information engineering from Beijing University of Aeronautics and Astronautics (Beihang University) in 2008 and the Ph.D. degree from Tsinghua University (THU), Beijing in 2013, both with the highest honors. During 2011-2012, he visited the Signals and Information Group (SIG) at Department of Electrical \& Computer Engineering (ECE) of University of Maryland (UMD), supported by China Scholarship Council (CSC) for one year.

Dr. Jiang is currently a research associate in ECE department of UMD with Prof. K. J. Ray Liu, and also a post-doctor in EE department of THU with Prof. Yong Ren. His research interests include the applications of game theory and queuing theory in wireless communication and networking and social networks.

Dr. Jiang received Best Paper Award from IEEE GLOBECOM in 2013, the Beijing Distinguished Graduated Student Award, Chinese National Fellowship and Tsinghua Outstanding Distinguished Doctoral Dissertation in 2013.

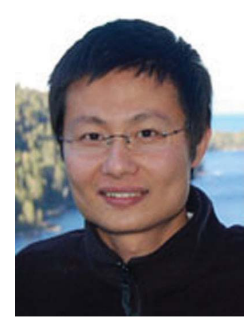

Yan Chen (S'06-M'11) received the Bachelors degree from University of Science and Technology of China in 2004, the M. Phil degree from Hong Kong University of Science and Technology (HKUST) in 2007, and the Ph.D. degree from University of Maryland College Park in 2011. His current research interests are in data science, network science, game theory, social learning and networking, as well as signal processing and wireless communications.

Dr. Chen is the recipient of multiple honors and awards including best paper award from IEEE GLOBECOM in 2013, Future Faculty Fellowship and Distinguished Dissertation Fellowship Honorable Mention from Department of Electrical and Computer Engineering in 2010 and 2011, respectively, Finalist of Deans Doctoral Research Award from A. James Clark School of Engineering at the University of Maryland in 2011, and Chinese Government Award for outstanding students abroad in 2011.

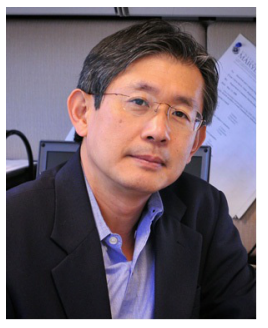

K. J. Ray Liu (F'03) was named a Distinguished Scholar-Teacher of University of Maryland, College Park, in 2007, where he is Christine Kim Eminent Professor of Information Technology. He leads the Maryland Signals and Information Group conducting research encompassing broad areas of signal processing and communications with recent focus on cooperative and cognitive communications, social learning and network science, information forensics and security, and green information and communications technology.

Dr. Liu is the recipient of numerous honors and awards including IEEE Signal Processing Society Technical Achievement Award and Distinguished Lecturer. He also received various teaching and research recognitions from University of Maryland including university-level Invention of the Year Award; and Poole and Kent Senior Faculty Teaching Award, Outstanding Faculty Research Award, and Outstanding Faculty Service Award, all from A. James Clark School of Engineering. An ISI Highly Cited Author, Dr. Liu is a Fellow of IEEE and AAAS.

Dr. Liu is Past President of IEEE Signal Processing Society where he has served as Vice President Publications and Board of Governor. He was the Editor-in-Chief of IEEE Signal Processing Magazine and the founding Editor-in-Chief of EURASIP Journal on Advances in Signal Processing. 